\title{
Clinical importance of testing for clopidogrel resistance in patients undergoing carotid artery stenting - a systematic review
}

\author{
Sabine L. Collette ${ }^{1}$, Reinoud P. H. Bokkers ${ }^{1}$, Rudi A. J. O. Dierckx ${ }^{1,2}$, Maarten J. van der Laan ${ }^{3}$, \\ Clark J. Zeebregts ${ }^{3}$, Maarten Uyttenboogaart ${ }^{1,4}$ \\ ${ }^{1}$ Department of Radiology, Medical Imaging Center, University Medical Center Groningen, University of Groningen, Groningen, The Netherlands; \\ ${ }^{2}$ Department of Nuclear Medicine and Molecular Imaging, Medical Imaging Center, University Medical Center Groningen, University of \\ Groningen, Groningen, The Netherlands; ${ }^{3}$ Division of Vascular Surgery, Department of Surgery, University Medical Center Groningen, University \\ of Groningen, Groningen, The Netherlands; ${ }^{4}$ Department of Neurology, University Medical Center Groningen, University of Groningen, \\ Groningen, The Netherlands \\ Contributions: (I) Conception and design: All authors; (II) Administrative support: SL Collette; (III) Provision of study materials or patients: SL \\ Collette, M Uyttenboogaart; (IV) Collection and assembly of data: SL Collette; (V) Data analysis and interpretation: SL Collette, M Uyttenboogaart, \\ RPH Bokkers; (VI) Manuscript writing: All authors; (VII) Final approval of manuscript: All authors. \\ Correspondence to: Sabine L. Collette, MD. University Medical Center Groningen, Huispostcode AB51, Hanzeplein 1, Postbus 30001, 9700 RB \\ Groningen, the Netherlands. Email: s.l.collette@umcg.nl.
}

\begin{abstract}
Dual antiplatelet therapy is frequently prescribed for patients undergoing carotid artery stenting (CAS), however clopidogrel resistance might cause thromboembolic complications. The role of testing for clopidogrel resistance in patients undergoing CAS is unclear. In this study, we aimed to review the periprocedural thromboembolic outcomes in clopidogrel resistant patients who underwent CAS. We conducted a review of PubMed, EMBASE, and the Cochrane Library up to October 7, 2020. Studies were included that investigated at least ten patients aged 18 years or older with a symptomatic carotid artery stenosis requiring CAS. Studies were excluded that investigated patients with a carotid artery dissection, case reports, case series of less than ten patients, reviews, commentaries, letters to the editors, and conference abstracts. The primary endpoint was the incidence of thromboembolic events. One hundred seventy-seven unique articles were identified of which three studies were included in our systematic review. The sample sizes ranged from 76 to 449 patients and the follow-up duration from 24 hours to 2 years postprocedural. Two retrospective observational studies determined clopidogrel resistance using measurement of P2Y12 reaction units, and one historical cohort study used genetic testing. Two studies concluded that clopidogrel resistance was a risk factor for thromboembolic complications, the other found higher values of P2Y12 reaction units in patients with thromboembolic events compared to those without. In conclusion, current literature supports a possible relationship between clopidogrel resistance and thromboembolic complications in patients who underwent CAS. Preprocedural testing for clopidogrel resistance might therefore be of additional value. Randomized studies using a valid, reliable clopidogrel resistance test and clinical endpoints, are however required to make a definitive statement and to determine the impact of the thromboembolic complications. This study was registered within PROSPERO (CRD42020197318).
\end{abstract}

Keywords: Carotid artery stenting (CAS); clopidogrel resistance; CYP2C19; pharmacogenetics; thromboembolic outcomes

Submitted Oct 28, 2020. Accepted for publication Feb 04, 2021.

doi: $10.21037 /$ atm-20-7153

View this article at: http://dx.doi.org/10.21037/atm-20-7153

(c) Annals of Translational Medicine. All rights reserved. 


\section{Introduction}

Carotid artery stenting (CAS) is a widely used treatment method for patients with a symptomatic or asymptomatic carotid artery stenosis (1). In the peri- and postprocedural phase, patients are at increased risk for thromboembolic complications, such as in-stent thrombosis or recurrent stroke (2-5). To reduce the risk of such events, treatment with clopidogrel and aspirin is frequently prescribed from approximately three days prior to CAS until at least thirty days postprocedural (1). Nevertheless, the intended treatment effect of this dual antiplatelet therapy is not always achieved despite patient adherence. This might be due to clopidogrel resistance $(6,7)$.

Clopidogrel is a pro-drug that is converted to an active metabolite in the liver by cytochrome P450 enzymes, in which CYP2C19 plays an important role $(8,9)$. The active metabolite binds irreversibly to the $\mathrm{P} 2 \mathrm{Y} 12$ receptor and consequently inhibits the binding of adenosine diphosphate. This leads to a decrease in platelet activation, platelet aggregation, and clot formation (9). In clopidogrel resistance, a relevant change in platelet function is absent despite adequate clopidogrel dosage. This is mostly caused by a CYP2C19 polymorphism $(10,11)$. From a pharmacological point of view, clopidogrel resistance can be delineated with a P2Y12 reaction units (PRU) value $\geq 235$ or percentage platelet inhibition $\leq 20 \%(7,12)$. Clinically, it can be observed by the development of thromboembolic complications despite clopidogrel treatment (13).

Associations between clopidogrel resistance and thromboembolic complications have been found repeatedly in patients who underwent a percutaneous coronary intervention and received clopidogrel (14). The role of clopidogrel resistance testing in patients undergoing CAS is however unclear (1). We therefore aimed to review the periprocedural thromboembolic outcomes in clopidogrel resistant patients who underwent CAS.

We present the following article in accordance with the PRISMA reporting checklist (available at http://dx.doi. org/10.21037/atm-20-7153).

\section{Methods}

\section{Search strategy}

To identify studies investigating the relationship between clopidogrel resistance and thromboembolic complications during and after CAS, we conducted a review of PubMed, EMBASE, and the Cochrane Library up to October 7,
2020. Our search string consisted of the following, fixed, structure: 'carotid artery stenting' AND 'clopidogrel' AND ('clopidogrel resistance' OR 'platelet function tests'). The search was completed with relevant synonyms (details can be found online in the Appendix 1).

The articles found by the search string were screened for eligibility by reading the title and abstract. If an article was considered potentially relevant, the full text version was assessed. Additional relevant publications were identified in two ways. First, for each article found on PubMed, the first 40 similar articles were screened for relevance after they were filtered on 'best match'. Second, references of the articles that were screened in whole were reviewed.

The selection of studies was performed independently by two authors (SC and MU). The electronic searches, identification of additional publications, and removal of duplicates was executed by one author (SC). Discrepancies were resolved by discussion.

The identification and removal of duplicates were performed using Endnote X9 software (Clarivate Analytics, Philadelphia, Pennsylvania, USA). Studies were screened using Covidence software (Veritas Health Innovation, Melbourne, Australia; available at www.covidence.org). This study is registered within PROSPERO (CRD42020197318).

\section{Study selection}

Studies were included that investigated at least ten patients aged 18 years or older with a symptomatic carotid artery stenosis requiring CAS. Excluded were studies investigating patients with a carotid artery dissection, case reports, case series of less than ten patients, reviews, commentaries, letters to the editors, and conference abstracts.

\section{Data collection}

After a study was included, the following data was collected: first author, year of publication, country of study, study design, sample size, mean age, comparison, type of clopidogrel resistance test, threshold used for the clopidogrel resistance test, number of clopidogrel resistant patients, duration of follow-up, primary endpoint, and number of patients with the primary endpoint.

\section{Quality assessment}

The risk of bias was determined using the 'Risk Of Bias In Non-randomized Studies of Interventions' (ROBINS-I) 


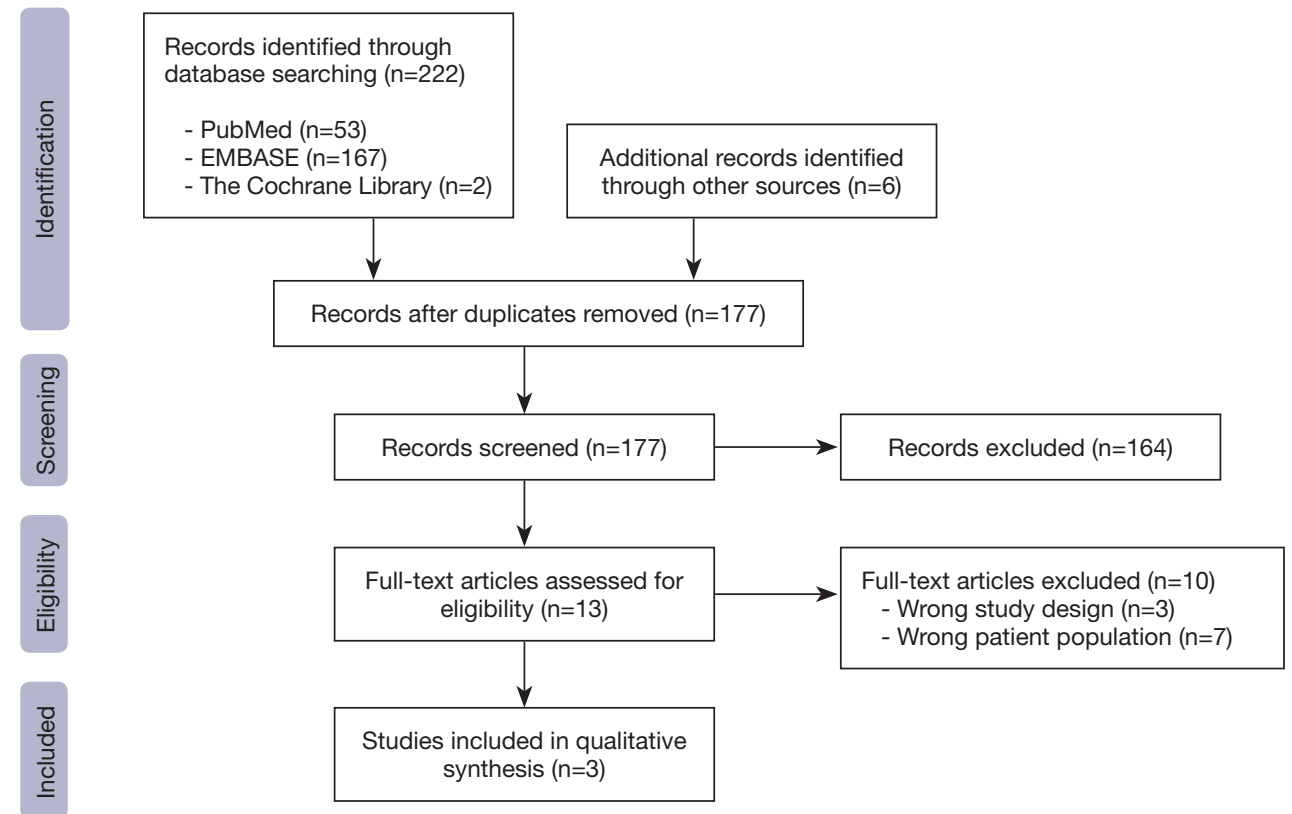

Figure 1 Flowchart of included studies.

tool. The assessment took place across seven domains, followed by an overall judgment (low, moderate, serious or critical risk of bias, or no information). A study with a low risk of bias was considered to be comparable to a randomized trial, and a study with a critical risk of bias was too questionable to include in the systematic review (15).

The strengths of the research methods and results were determined using 'Methodological Index for NonRandomized Studies' (MINORS). This tool consists of twelve items that are scored with 0 (not reported), 1 (reported but inadequate) or 2 (reported and adequate) points. A total score of 16 points was considered ideal for non-comparative studies, whereas a total score of 24 points was considered ideal for comparative studies (16).

Two authors (SC and MU) independently assessed the quality of the studies. Disagreement was solved by discussion.

\section{Outcomes}

The primary endpoint was the incidence of thromboembolic events.

\section{Results}

\section{Search results}

The search yielded 53 articles from PubMed, 167 articles from EMBASE, 2 articles from the Cochrane Library, and 6 articles through other sources. Following the removal of duplicates, 177 unique articles remained. One hundred sixty-four were excluded during screening for title and abstract, and 10 during the full text review. This left 3 articles to be included for our systematic review (Figure 1).

\section{Quality assessment}

All studies were included in the systematic review as their risk of bias was not considered to be critical (Table 1). In the assessment of methodological quality, none of the studies received the maximum MINORS score. For the noncomparative study (18), the MINORS score was 8 . The items that were not adequately reported, and consequently received a score of 0 points, include a prospective collection of the data, unbiased assessment of the study endpoints, loss to follow up less than $5 \%$, and prospective calculation of the study size. For the comparative studies, the MINORS scores were 17 (17) and 18 (19). The items of the weakest reporting were almost consistent with those of the non-comparative study. The main difference was the sufficient documentation of the loss to follow-up in the comparative studies (Table 2).

\section{Characteristics}

Two of the eligible studies were retrospective observational 
Table 1 'Risk Of Bias In Non-randomized Studies of Interventions' (ROBINS-I) assessment of the included studies

\begin{tabular}{|c|c|c|c|c|c|c|c|c|}
\hline Study & $\begin{array}{c}\text { Baseline } \\
\text { confounding }\end{array}$ & $\begin{array}{l}\text { Selection of } \\
\text { participants }\end{array}$ & $\begin{array}{l}\text { Classification of } \\
\text { interventions }\end{array}$ & $\begin{array}{l}\text { Deviation from } \\
\text { intended interventions }\end{array}$ & $\begin{array}{l}\text { Missing } \\
\text { data }\end{array}$ & $\begin{array}{l}\text { Measurement } \\
\text { of outcomes }\end{array}$ & $\begin{array}{l}\text { Selection of the } \\
\text { reported result }\end{array}$ & Overall \\
\hline $\begin{array}{l}\text { Song et al. } \\
\text { (17) [2013] }\end{array}$ & Moderate & Moderate & Low & Low & Low & Low & Low & Moderate \\
\hline $\begin{array}{l}\text { Sorkin et al. } \\
\text { (18) [2014] }\end{array}$ & Serious & Moderate & Moderate & Low & Moderate & Low & Serious & Serious \\
\hline
\end{tabular}

Critical, critical risk of bias; low, low risk of bias; moderate, moderate risk of bias; serious, serious risk of bias.

Table 2 'Methodological Index for Non-Randomized Studies' (MINORS) scores of the included studies

\begin{tabular}{|c|c|c|c|}
\hline Items & Song et al. (18) [2013] & Sorkin et al. (17) [2014] & Zhu et al. (19) [2016] \\
\hline 2. Inclusion of consecutive patients & 2 & 2 & 2 \\
\hline 3. Prospective collection of data & 0 & 0 & 0 \\
\hline 4. Endpoints appropriate to the aim of the study & 2 & 2 & 2 \\
\hline 6. Follow-up period appropriate to the aim of the study & 1 & 2 & 2 \\
\hline 7. Loss to follow-up less than $5 \%$ & 2 & 0 & 2 \\
\hline 8. Prospective calculation of the study size & 0 & 0 & 0 \\
\hline \multicolumn{4}{|l|}{ Additional criteria for comparative studies } \\
\hline 11. Baseline equivalence of groups & 2 & & 2 \\
\hline 12. Adequate statistical analyses & 2 & & 2 \\
\hline Total MINORS score [maximum possible score] & $17[24]$ & 8 [16] & 18 [24] \\
\hline
\end{tabular}

Each item is scored with 0 (not reported), 1 (reported but inadequate) or 2 (reported and adequate) points. The total score that was considered ideal was 16 points for non-comparative studies and 24 points for comparative studies.

studies $(17,18)$ and one was a historical cohort study (19). They were performed in South Korea (17), United States of America (18), and China (19). Sample sizes ranged from 76 to 449 patients. Overall, the studies aimed at investigating the relationship of clopidogrel resistance or PRU values with thromboembolic outcomes after CAS. The primary endpoints included new cerebral ischemic lesions observed on brain magnetic resonance imaging (MRI) (17), (ipsilateral) ischemic stroke, (ipsilateral) transient ischemic attack $(18,19)$, previous ischemic symptom recurrence, stent thrombosis, and death (19) (Table 3).

\section{Clopidogrel resistance testing and outcome}

Tests that were used to determine clopidogrel resistance varied among the included studies. Measurement of PRU values was used in the studies of Song et al. (17) and Sorkin et al. (18); genetic testing was performed in the article of Zhu et al. (19).

\section{Measurement of PRU values}

PRU values can be measured with a point-of-care assay 


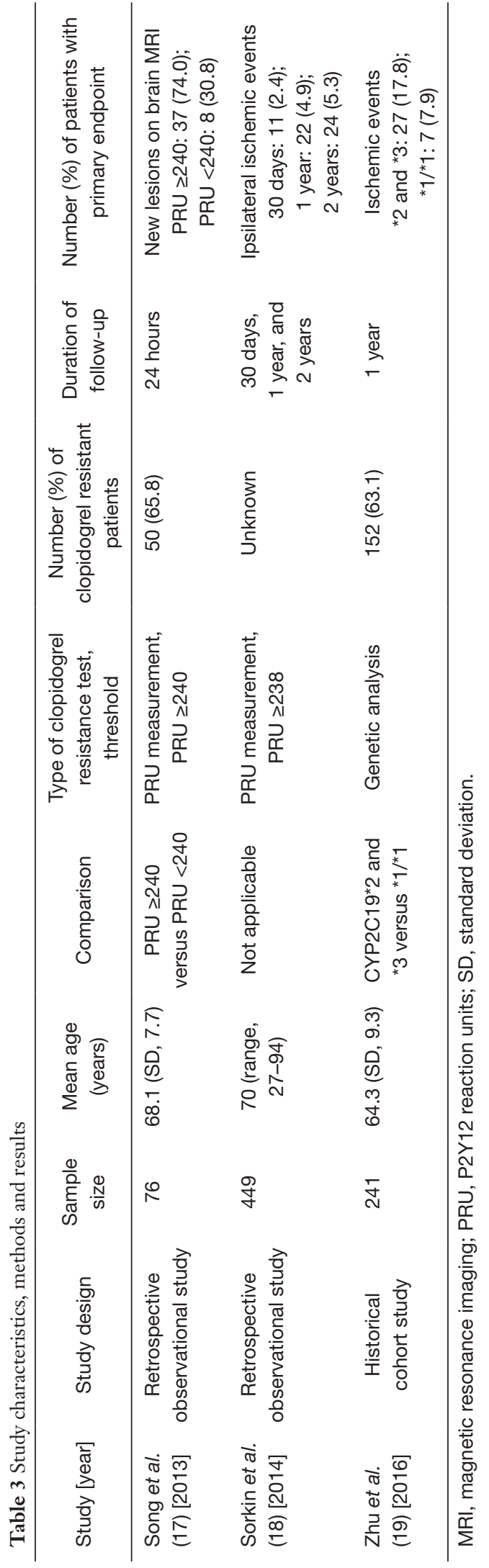

able to rapidly test the platelet activity of patients who are treated with clopidogrel. To perform the test, a small amount of whole-blood is required. In the test device, the blood sample is mixed with adenosine diphosphate and prostaglandin E1 to induce platelet aggregation. During the process of platelet aggregation, an amount of light transmitted through the sample is measured. If platelet aggregation is not adequately inhibited by clopidogrel, the sample will be clearer which results in a higher absorbance of light. The amount of platelet aggregation mediated by the $\mathrm{P} 2 \mathrm{Y} 12$ receptor is expressed as PRU, with high PRU values indicating more reactive platelets $(20,21)$. Although an optimal threshold is under debate (22-24), a PRU value $\geq 235$ or percentage platelet inhibition $\leq 20 \%$ are generally considered clopidogrel resistant $(7,12)$. In the studies of Song et al. (17) and Sorkin et al. (18), PRU values were determined using VerifyNow ${ }^{\circledR}$ (Accumetrics, San Diego, California, USA).

Song et al. (17) analyzed the relationship between clopidogrel resistance and new cerebral ischemic lesions detected on postprocedural brain MRI. A total of 50 out of the $76(65.8 \%)$ included patients had an increased PRU value. At 24 hours after CAS, the images showed that 45 (59.2\%) patients had newly developed cerebral ischemic lesions. Thirty-seven $(82.2 \%)$ of the patients with new ischemic lesions were clopidogrel resistant. In multivariate analysis, clopidogrel resistance was found to be associated with new ischemic events (OR, 6.804; 95\% CI, 2.22520.806).

Sorkin et al. (18) investigated whether ipsilateral cerebral ischemic events after CAS were related to PRU values. During follow-up at one and two years after CAS, the mean PRU values were found to be significantly higher in patients with an ipsilateral ischemic event than in patients without [PRU value at one year: 252 and $202(\mathrm{P}=0.008)$, respectively; PRU at two years: 244 and $203(\mathrm{P}=0.047)$, respectively]. The ischemic free survival did not differ between patients under and above the initial PRU threshold of $\geq 238$. When it was compared with a lower threshold, the ischemic free survival was longer for patients with a PRU value $\leq 198$ than for patients $\leq 237(\mathrm{P}=0.027)$.

\section{Genetic testing}

Important loss-of-function alleles are $\mathrm{CYP} 2 \mathrm{C} 19^{*} 2$ and CYP2C19*3. These allelic variants are associated with a reduced activity of the CYP2C19 enzyme, which keeps the amount of active clopidogrel metabolite low $(11,25,26)$. 
Therefore, the risk of thromboembolic complications persists. In the study of Zhu et al. (19), CYP2C19*2 and CYP2C19*3 polymorphisms were detected by analyzing the images of the hybrid DNA sections obtained through polymerase chain reaction. The genetic analysis was performed using a commercially available kit (BaiO Technology Co, Ltd., Shanghai, China).

Zhu et al. (19) aimed to determine the relationship between the loss-of-function alleles CYP2C19*2 and *3 and the clinical efficacy of clopidogrel. A total of 152 out of the $241(63.1 \%)$ included patients had the CYP2C19*2 and *3 alleles. Ischemic events occurred in $27(17.8 \%)$ carriers of these alleles. Compared with the non-carriers, patients with the CYP2C19*2 and *3 alleles were found to be at increased risk of ischemic complications (hazard ratio, 2.131; 95\% CI, 1.067-4.255). No difference was observed between CYP2C19*2 and CYP2C19*3 carriers related to number of ischemic events. The risk of cumulative ischemic events was increased for patients with two CYP2C19*2 or CYP2C19*3 alleles compared to carriers of one allele (hazard ratio, 6.176; 95\% CI, 1.145-33.32).

\section{Discussion}

This systematic review included two retrospective observational studies and one historical cohort study, which investigated the relationship of clopidogrel resistance or PRU values with thromboembolic outcomes after CAS. Although different tests were used to diagnose clopidogrel resistance, two studies concluded that patients with clopidogrel resistance are more likely to experience thromboembolic events $(18,19)$. The other study found higher PRU values in patients with ischemic events at one and two years of follow-up, and a longer ischemic free survival in patients with a PRU value under the threshold of $\leq 198$ instead of the initial PRU threshold of $\leq 237$ (18).

These results are in line with other neuro-interventional studies in different populations $(7,27)$. In a 4.5 -year prospective study among 96 patients who underwent extracranial or intracranial neurovascular stenting, clopidogrel resistance (defined as percentage platelet inhibition $\leq 20 \%$, determined using VerifyNow ${ }^{\circledR}$ ) was associated with periprocedural thromboembolic complications. In addition, patients were divided in two groups: one group consisted of 49 patients of whom $33 \%$ had a percentage platelet inhibition $\leq 20 \%$, the other group consisted of 47 patients of whom $40 \%$ had a percentage platelet inhibition $\leq 20 \%$. In the group of 47 patients, additional clopidogrel was given to the clopidogrel resistant patients. This did however not result in a lower complication rate compared with the clopidogrel resistant patients whose dose of clopidogrel was not adjusted (7). A prospective study analyzed the mean percentage platelet inhibition (determined using VerifyNow ${ }^{\circledR}$ ) of 5 patients with and 48 patients without thromboembolic complications within six months after neurovascular stenting. A threshold of $<40 \%$ was defined as clopidogrel resistance. The mean platelet inhibition percentages differed significantly among both groups [patients with complications, 23\% (range, 21-27); without complications, 35\% (range, 16-91); $\mathrm{P}=0.01$ ] (27).

The clopidogrel metabolism was investigated by measuring PRU values or genetic testing. The advantages of measuring PRU values are that it is easy to perform, patients can be rapidly tested at bedside, and blood samples do not need any preparation before testing. Disadvantages are the relatively high costs compared with other platelet function assays and the variability of test results within an individual. The advantages of genetic testing are the consistent test results as a patient its genotype does not change over time and that testing can take place before treatment with clopidogrel. Disadvantages are the process of testing takes time, qualified personnel is needed to prepare the blood samples, the high costs in comparison with PRU measurement, and the impossibility to test patient adherence (insufficient clopidogrel intake would lead to abnormal PRU values, while genetic testing would not show any abnormalities). In clinical practice, measurement of PRU values might be more convenient than genetic testing because it can be performed in a short amount of time by a variety of health care workers.

There is an ongoing debate regarding the optimal threshold for the PRU values (22-24). This is reflected by the different PRU values $(\geq 240, \geq 238$, and $\geq 199)$ that were maintained in two of the included studies $(17,18)$. The most commonly used PRU value to define clopidogrel resistance, $\geq 235$, could be questioned as it is based on a relatively small sample size. In addition, the possible individual variability of PRU values during clopidogrel treatment was not taken into account during the determination of this threshold $(12,23,28)$. Among patients who underwent percutaneous coronary intervention, it was found that, even with a clopidogrel loading dose of $600 \mathrm{mg}$, PRU levels decreased during the first month of clopidogrel treatment. This result can be considered clinically relevant as the classification of the majority of patients with a PRU decrease changed from poor (PRU value 2235 ) to full responder (PRU value 
$<235)$. The PRU values did not differ between the next five months of follow-up (23). As in the included studies, clopidogrel treatment is usually started within three to seven days before the CAS, or in some cases only one day in advance, and continued for, preferably, at least thirty days (1,17-19). For the measurement of PRU values, blood samples are mostly drawn once and within two days before or after the CAS $(7,17,18,27)$. As PRU values can still lower within the first month of clopidogrel treatment, it could be hypothesized that treatment should start at least one month before CAS. Whether this early start of clopidogrel treatment outweighs the risk of bleeding in all types of responders and symptomatic patients should be investigated.

In addition to clopidogrel, patients of the included studies received aspirin from approximately seven days before the CAS until preferably thirty days postprocedural. As aspirin inhibits platelet activity through a different pathway than clopidogrel, it might have lowered the risk of thromboembolic complications (29). As consequence, a possible relationship of clopidogrel resistance or PRU values with thromboembolic complications could have been missed. The included studies showed however still significant results. Even if no significant results were found, the clinical implication could be questioned because dual antiplatelet therapy is prescribed to the majority of patients (1). Furthermore, two of the included studies also investigated the influence of aspirin resistance or assays and found no relationship with thromboembolic outcomes $(17,18)$. Although it has been found that aspirin resistant patients do respond less to clopidogrel than aspirin sensitive patients, which was also observed by Song et al. (17), it does not apply the other way around (30).

Although the incidence of clopidogrel resistance could not be precisely determined due to the use of various tests and thresholds, it appears to be common in patients undergoing CAS [65.8\% in the study of Song et al. (17) and $63.1 \%$ in the study of Zhu et al. (19)]. Follow-up research into optimization of antiplatelet treatment for this population seems therefore to be warranted. In an observational study amongst 77 patients, the percentage platelet inhibition was compared between patients who were treated with clopidogrel and cilostazol (15 patients) or clopidogrel alone (62 patients) for CAS, acute ischemic stroke or transient ischemic attack. Clopidogrel resistance (defined as percentage platelet inhibition $<20 \%$, determined using VerifyNow ${ }^{\circledR}$ ) was found in none of the patients treated with clopidogrel and cilostazol, and in 18 out of the
$62(29 \%)$ patients treated with clopidogrel alone. Moreover, the percentage platelet inhibition was significantly higher in patients who received additionally cilostazol [cilostazol and clopidogrel: mean, 64.9\%; standard deviation (SD), 22.7; clopidogrel only: mean, 41.7\%; SD, 28.0, $\mathrm{P}=0.005]$. This might indicate that the addition of cilostazol increased the percentage platelet inhibition, however, no definite conclusions could be drawn due to potential selection bias and inclusion of a limited number of cilostazol-treated patients (31). A similar effect of cilostazol was found in a prospective non-randomized study amongst 137 patients who underwent CAS. A significantly lower PRU value (determined using VerifyNow ${ }^{\circledR}$ ) was detected in patients treated with cilostazol and clopidogrel than in patients treated with aspirin and clopidogrel (mean, 147.0; SD, 64.3, and mean, 235.4; SD 71.2, respectively). Moreover, the incidence of new ischemic ipsilateral lesions was significantly lower in patients who received cilostazol and the incidence of hemorrhagic events did not significantly differ between both groups. This might suggest that cilostazol is effective and safe, however, as for the previous study, the results might have been affected by selection bias due to the nonrandomized design (32). Another treatment option that has been investigated is ticlopidine/ginkgo biloba. In a multicenter, randomized, controlled trial, clopidogrel resistant patients (defined as percentage platelet inhibition $<20 \%$, determined using VerifyNow ${ }^{\circledR}$ ) who underwent CAS were randomized into the ticlopidine/ginkgo biloba arm or clopidogrel arm. During the first interim analysis amongst 42 patients (20 and 22 patients, respectively), it was decided to terminate the trial early due to an impractical recalculated sample size. At this stage, the incidence of new ischemic lesions at 24 hours after CAS did not differ between both groups, although treatment with ticlopidine/ginkgo biloba increased the PRU values significantly [difference, 21.0 (6.0-35.0) and $0.0(-3.0-3.0), \mathrm{P}<0.001]$ (33). Based on the aforementioned studies, a combination of cilostazol and clopidogrel might be promising in prevention of thromboembolic complications in clopidogrel resistant patients who undergo CAS. Randomized clinical trials are needed to further elaborate on the effectiveness and safety of cilostazol.

This systematic review has some limitations. First, only three studies were identified due to the limited availability of publications. In addition, the studies that were included used different tests for clopidogrel resistance and various thresholds. Although the results pointed in the same direction, no definitive statement could be made 
about the possible relationship clopidogrel resistance and thromboembolic complications in patients who underwent CAS. Second, the included studies were (partly) retrospective in design, which makes them susceptible to baseline confounding and missing data. This may have affected the results in unpredictable ways. Third, the in- and exclusion criteria of all studies might have caused selection bias with the likely direction in favor of the patients without complications. Nevertheless, the studies identified clopidogrel resistance or higher PRU values as a risk factor for thromboembolic complications. In addition, two studies were performed among Asians, who are known to have the highest prevalence of clopidogrel resistance (34-36). This could have resulted in a stronger significance compared with similar studies in patients with a different racial background. Fourth, the outcome assessors of the included studies were not blinded to the intervention and outcomes. The risk of bias was however expected to be low because both test results and thromboembolic complications were objective measurements. Final, the impact of thromboembolic events could not be adequately determined as only one clinical endpoint (death) was examined.

\section{Conclusions}

Current literature supports a possible relationship between clopidogrel resistance and thromboembolic complications in patients who underwent CAS. Preprocedural testing for clopidogrel resistance might therefore be of additional value. Randomized studies using a valid, reliable clopidogrel resistance test and clinical endpoints, are however required to make a definitive statement and to determine the impact of the thromboembolic complications.

\section{Acknowledgments}

Funding: None.

\section{Footnote}

Provenance and Peer Review: This article was commissioned by the Guest Editor (Kosmas I. Paraskevas) for the series "Carotid Artery Stenosis and Stroke - Prevention and Treatment Part II" published in Annals of Translational Medicine. The article has undergone external peer review.

Reporting Checklist: The authors have completed the PRISMA reporting checklist. Available at http://dx.doi. org/10.21037/atm-20-7153

Conflicts of Interest: All authors have completed the ICMJE uniform disclosure form (available at http://dx.doi. org/10.21037/atm-20-7153). The series "Carotid Artery Stenosis and Stroke - Prevention and Treatment Part II" was commissioned by the editorial office without any funding or sponsorship. The authors have no other conflicts of interest to declare.

Ethical Statement: The authors are accountable for all aspects of the work in ensuring that questions related to the accuracy or integrity of any part of the work are appropriately investigated and resolved.

Open Access Statement: This is an Open Access article distributed in accordance with the Creative Commons Attribution-NonCommercial-NoDerivs 4.0 International License (CC BY-NC-ND 4.0), which permits the noncommercial replication and distribution of the article with the strict proviso that no changes or edits are made and the original work is properly cited (including links to both the formal publication through the relevant DOI and the license). See: https://creativecommons.org/licenses/by-nc-nd/4.0/.

\section{References}

1. Naylor AR, Ricco JB, de Borst GJ, et al. Editor's Choice - Management of Atherosclerotic Carotid and Vertebral Artery Disease: 2017 Clinical Practice Guidelines of the European Society for Vascular Surgery (ESVS). Eur J Vasc Endovasc Surg 2018;55:3-81.

2. Brott TG, Hobson RW 2nd, Howard G, et al. Stenting versus endarterectomy for treatment of carotid-artery stenosis. N Engl J Med 2010;363:11-23.

3. Ederle J, Dobson J, Featherstone RL, et al. Carotid artery stenting compared with endarterectomy in patients with symptomatic carotid stenosis (International Carotid Stenting Study): an interim analysis of a randomised controlled trial. Lancet 2010;375:985-97.

4. Eckstein HH, Ringleb P, Allenberg JR, et al. Results of the Stent-Protected Angioplasty versus Carotid Endarterectomy (SPACE) study to treat symptomatic stenoses at 2 years: a multinational, prospective, randomised trial. Lancet Neurol 2008;7:893-902.

5. Mas JL, Chatellier G, Beyssen B, et al. Endarterectomy versus stenting in patients with symptomatic severe carotid stenosis. N Engl J Med 2006;355:1660-71. 
6. Gurbel PA, Tantry US. Do platelet function testing and genotyping improve outcome in patients treated with antithrombotic agents?: platelet function testing and genotyping improve outcome in patients treated with antithrombotic agents. Circulation 2012;125:1276-87; discussion 87.

7. Fifi JT, Brockington C, Narang J, et al. Clopidogrel resistance is associated with thromboembolic complications in patients undergoing neurovascular stenting. AJNR Am J Neuroradiol 2013;34:716-20.

8. Kazui M, Nishiya Y, Ishizuka T, et al. Identification of the human cytochrome P450 enzymes involved in the two oxidative steps in the bioactivation of clopidogrel to its pharmacologically active metabolite. Drug Metab Dispos 2010;38:92-9.

9. Trenk D, Hochholzer W. Genetics of platelet inhibitor treatment. Br J Clin Pharmacol 2014;77:642-53.

10. Nguyen TA, Diodati JG, Pharand C. Resistance to clopidogrel: a review of the evidence. J Am Coll Cardiol 2005;45:1157-64.

11. Zhuo ZL, Xian HP, Long Y, et al. Association between CYP2C19 and ABCB1 polymorphisms and clopidogrel resistance in clopidogrel-treated Chinese patients. Anatol J Cardiol 2018;19:123-9.

12. Price MJ, Endemann S, Gollapudi RR, et al. Prognostic significance of post-clopidogrel platelet reactivity assessed by a point-of-care assay on thrombotic events after drugeluting stent implantation. Eur Heart J 2008;29:992-1000.

13. Stone GW, Witzenbichler B, Weisz G, et al. Platelet reactivity and clinical outcomes after coronary artery implantation of drug-eluting stents (ADAPT-DES): a prospective multicentre registry study. Lancet 2013;382:614-23.

14. Aradi D, Kirtane A, Bonello L, et al. Bleeding and stent thrombosis on P2Y12-inhibitors: collaborative analysis on the role of platelet reactivity for risk stratification after percutaneous coronary intervention. Eur Heart J 2015;36:1762-71.

15. Sterne JA, Hernan MA, Reeves BC, et al. ROBINS-I: a tool for assessing risk of bias in non-randomised studies of interventions. BMJ 2016;355:i4919.

16. Slim K, Nini E, Forestier D, et al. Methodological index for non-randomized studies (minors): development and validation of a new instrument. ANZ J Surg 2003;73:712-6.

17. Song TJ, Suh SH, Min PK, et al. The influence of anti-platelet resistance on the development of cerebral ischemic lesion after carotid artery stenting. Yonsei Med J 2013;54:288-94.
18. Sorkin GC, Dumont TM, Wach MM, et al. Carotid artery stenting outcomes: do they correlate with antiplatelet response assays? J Neurointerv Surg 2014;6:373-8.

19. Zhu WY, Zhao T, Xiong XY, et al. Association of CYP2C19 Polymorphisms with the Clinical Efficacy of Clopidogrel Therapy in Patients Undergoing Carotid Artery Stenting in Asia. Sci Rep 2016;6:25478.

20. Malinin A, Pokov A, Spergling M, et al. Monitoring platelet inhibition after clopidogrel with the VerifyNow-P2Y12(R) rapid analyzer: the VERIfy Thrombosis risk ASsessment (VERITAS) study. Thromb Res 2007;119:277-84.

21. van Werkum JW, Harmsze AM, Elsenberg EH, et al. The use of the VerifyNow system to monitor antiplatelet therapy: a review of the current evidence. Platelets 2008;19:479-88.

22. Bender MT, Zarrin DA, Campos JK, et al. Precision of VerifyNow P2Y12 Assessment of Clopidogrel Response in Patients Undergoing Cerebral Aneurysm Flow Diversion. Neurosurgery 2019;85:543-9.

23. Campo G, Parrinello G, Ferraresi P, et al. Prospective evaluation of on-clopidogrel platelet reactivity over time in patients treated with percutaneous coronary intervention relationship with gene polymorphisms and clinical outcome. J Am Coll Cardiol 2011;57:2474-83.

24. Kirtane AJ, Parikh PB, Stuckey TD, et al. Is There an Ideal Level of Platelet P2Y12-Receptor Inhibition in Patients Undergoing Percutaneous Coronary Intervention?: "Window" Analysis From the ADAPTDES Study (Assessment of Dual AntiPlatelet Therapy With Drug-Eluting Stents). JACC Cardiovasc Interv 2015;8:1978-87.

25. Collet JP, Hulot JS, Pena A, et al. Cytochrome P450 2C19 polymorphism in young patients treated with clopidogrel after myocardial infarction: a cohort study. Lancet 2009;373:309-17.

26. Pan Y, Chen W, Xu Y, et al. Genetic Polymorphisms and Clopidogrel Efficacy for Acute Ischemic Stroke or Transient Ischemic Attack: A Systematic Review and MetaAnalysis. Circulation 2017;135:21-33.

27. Ryu DS, Hong CK, Sim YS, et al. Anti-platelet drug resistance in the prediction of thromboembolic complications after neurointervention. J Korean Neurosurg Soc 2010;48:319-24.

28. Hochholzer W, Ruff CT, Mesa RA, et al. Variability of individual platelet reactivity over time in patients treated with clopidogrel: insights from the ELEVATE-TIMI 56 trial. J Am Coll Cardiol 2014;64:361-8.

29. Hankey GJ, Eikelboom JW. Aspirin resistance. Lancet 
2006;367:606-17.

30. Lev EI, Patel RT, Maresh KJ, et al. Aspirin and clopidogrel drug response in patients undergoing percutaneous coronary intervention: the role of dual drug resistance. J Am Coll Cardiol 2006;47:27-33.

31. Maruyama H, Takeda H, Dembo T, et al. Clopidogrel resistance and the effect of combination cilostazol in patients with ischemic stroke or carotid artery stenting using the VerifyNow P2Y12 Assay. Intern Med 2011;50:695-8.

32. Nakagawa I, Park HS, Wada T, et al. Efficacy of cilostazolbased dual antiplatelet treatment in patients undergoing carotid artery stenting. Neurol Res 2017;39:695-701.

33. Chung JW, Kim SJ, Hwang J, et al. Comparison of

Cite this article as: Collette SL, Bokkers RPH, Dierckx RAJO, van der Laan MJ, Zeebregts CJ, Uyttenboogaart M. Clinical importance of testing for clopidogrel resistance in patients undergoing carotid artery stenting-a systematic review. Ann Transl Med 2021;9(14):1211. doi: 10.21037/atm-20-7153
Clopidogrel and Ticlopidine/Ginkgo Biloba in Patients With Clopidogrel Resistance and Carotid Stenting. Front Neurol 2019;10:44.

34. Hasan MS, Basri HB, Hin LP, et al. Genetic polymorphisms and drug interactions leading to clopidogrel resistance: why the Asian population requires special attention. Int J Neurosci 2013;123:143-54.

35. Jiang XL, Samant S, Lesko LJ, et al. Clinical pharmacokinetics and pharmacodynamics of clopidogrel. Clin Pharmacokinet 2015;54:147-66.

36. Brown SA, Pereira N. Pharmacogenomic Impact of CYP2C19 Variation on Clopidogrel Therapy in Precision Cardiovascular Medicine. J Pers Med 2018;8:8. 